\title{
A STRAIGHTFORWARD APPROACH TO THE DERIVATION OF TOPOLOGIES
}

\author{
Garrelts, Enno; Roth, Daniel; Binz, Hansgeorg \\ University of Stuttgart
}

\begin{abstract}
The design of topologically optimized structures is straightforward, although the main problem is really to derive the correct structure in each instance. During the development of structures for additive manufacturing in particular, saving weight is crucial because weight is proportional to cost.

In this contribution firstly, different approaches to topological optimization are presented and discussed. While computer-assisted tools provide high accuracy and demand defined conditions, approaches utilizing a pen and paper can be conducted relatively quickly, although these only provide little guidance.

Secondly, a new approach is presented which is advantageous with regard to effort and affordability, yet which maintains an accuracy of results. To support the designer, an artificial neural network is trained to adapt suitable Michell structures within a given design area. These structures depict optimal paths to conduct the loads through a component and provide guidance in designing an appropriate topology.

Evaluation has demonstrated that this new approach is capable of supporting designers in achieving lightweight structures.
\end{abstract}

Keywords: Additive Manufacturing, Lightweight design, App, Early design phases, Artificial intelligence

\section{Contact:}

Garrelts, Enno

University of Stuttgart

Institute for Engineering Design and Industrial Design, Graduate School of Excellence advanced Manufacturing Engineering

Germany

enno.garrelts@iktd.uni-stuttgart.de

Cite this article: Garrelts, E., Roth, D., Binz, H. (2019) 'A Straightforward Approach to the Derivation of Topologies', in Proceedings of the 22nd International Conference on Engineering Design (ICED19), Delft, The Netherlands, 5-8 August 2019. DOI:10.1017/dsi.2019.272 


\section{INTRODUCTION}

The generic term "additive manufacturing" encompasses various processes that manufacture components layer by layer. Selective Laser Melting (SLM) is an additive process for the production of metal parts already employed in series production (Wohlers, 2017). In SLM, a metal powder is melted locally in a powder bed by a laser. This manufacturing method affords great freedom of design, thus enabling products and components to satisfy requirements in new and sometimes better ways (Gebhardt, 2016). The main obstacles to the further spread of SLM are currently the high manufacturing costs (acatech Deutsche Akademie der Technikwissenschaften, 2016). Due to the manufacturing principle, these are directly related to the component volume, or rather its weight (Gebhardt, 2016). Strategies for weight reduction and consecutively manufacturing cost reduction gain a higher status.

\subsection{Motivation}

One effective way to reduce the weight of components is to optimize the topology of the structure. Support for designers in this optimization can be divided into two categories: sketch-based support and computerbased mathematical support. The former includes methods such as "thinking tools after nature" (Mattheck, 2010). These do not require a high detail of design as input, yet also yield results of relatively low detail. In comparison to computer-based mathematical support, sketch-based support is relatively fast, but also requires several minutes for one design version. The mathematical approaches require a high detail of design for optimization and are relatively slow. However, they provide highly detailed results.

In the late concept phase or the early embodiment design phase of product development, designers are tasked with realizing lightweight structures. In these early design stages, few details are defined, and several variants can be developed simultaneously at a component level. The variants vary in terms of geometrical dimensions, location of bearings, angles of applied forces and various other properties. Any support provided in early stages should be time-efficient and comprehensible; in other words, more like the "thinking tools after nature" than the mathematical support methods (Keller and Binz, 2009; Posner et al., 2012). The development of multiple alternatives causes the time required for optimization to add up. Since time is an important measure for development (Pahl et al., 2007), this time should be reduced to a minimum. Comprehensibility is also important since the designer has several tasks in mind simultaneously. The less effort it takes to derive the topology, the better, since support methods whose application is complex are often neglected.

\subsection{Problem statement}

Sketch-based support requires the designer to concentrate on the topology and necessitates some training. Even for a trained designer, several minutes are required for deriving an appropriate topology (Posner et al., 2014; Mattheck, 2010). The mathematical solutions are even more complicated: Here, lengthy training is required in order to deal with the complexity involved. In addition, anything from several minutes to hours is required for deriving an appropriate, topologically optimized design.

The time consumption and the level of complexity of applying a support method involved in deriving a topology are compared in Figure 1. A support method for the early stages of design - located in the lower left-hand corner of the graph - would be preferable. An investigation is to be conducted to determine which approach can produce results with fuzzy and low-quality input in a highly time-efficient manner.

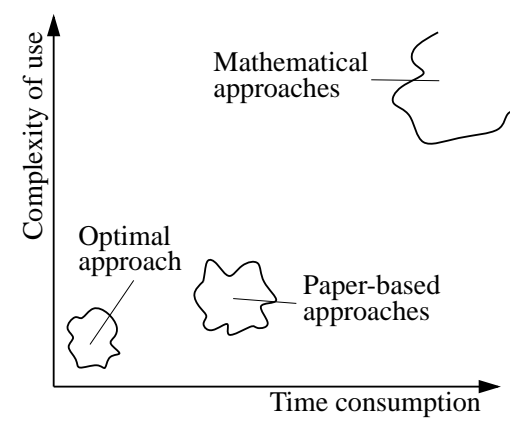

Figure 1: Schematic classification of methods used for topological optimization 
In recent years, artificial neural networks have become a popular solution in informatics for problems with inaccurate and fuzzy inputs (Aggarwal, 2018). One example of their application in a classification task is the recognition of handwritten characters. Recent studies have also shown how to utilize this technology to generate new pictures (Isola et al., 2016). These networks are capable of solving tasks formulated in a fuzzy manner in a very short time. The properties of artificial neural networks make them one possible basis for a new and faster support method for deriving topologies.

\subsection{Objective}

Since even the sketch-based approaches to the derivation of topologies have deficits, the following research question arises for this paper: How can designers derive topologies in a time-efficient and comprehensible way in the late concept phases of design? The possibilities and deficits known approaches demonstrate are to be clarified. The hypothesis of this paper is that a new method supported by an artificial neural network could provide designers with very rapid assistance with design proposals. The fundamental applicability is also to be tested.

If the new support approach proves promising, its use could result in topologies that achieve a sufficient detail, meaning that the designer could forego computer-based mathematical topology optimization in certain cases. Such cases potentially include the development of design objects. In the case of components requiring extreme lightweight design, the support tool could help in selecting the best possible variants for subsequent, detailed optimization. By proceeding in this way, it would be possible to pursue more promising approaches before optimizing only the best ones.

\subsection{Structure}

Section 2 presents the different approaches that designers can apply to derive an optimized topology of components. This paper therefore distinguishes between methods that are mathematical and those that can be carried out merely with a pen and paper. Subsequently, the deficits of these approaches are summarized. Section 3 presents a new approach, for which the operating principle of artificial neural networks (which might assist in the realization of a support tool) is presented. The procedure of employing the new approach is subsequently explained. To evaluate the new approach, three brief evaluations of its basic feasibility and support value are conducted in Section 4, after which Section 5 discusses the results of this evaluation before a brief conclusion in Section 6 .

\section{DERIVATION OF TOPOLOGIES}

In the search for an optimization of geometries during the design process, it is necessary to clarify precisely what the objective of the optimization is. According to Michell, an optimal structure is one that realizes a volume minimum within the framework of the permitted paths (Michell, 1904).

\subsection{Optimal topologies}

In addition to the definition of optimality above, one of the findings of Michell is that a truss structure achieves the best possible material utilization when all its elements exhibit either maximum tensile or maximum compressive strain. With regard to tension and pressure alone, the material utilization in each element of these structures is $100 \%$. The results of his work are best shown in truss structures, for which he deduces (based on these considerations) that an optimal structure follows a system of trajectories, which are orthogonal regardless of the load (Michell, 1904). Such a structure is therefore the system with the highest stiffness at a given weight, or the structure with the lowest weight at a given stiffness. The considerations focus more on an idealized optimum than on direct applicability. The Michell structures describe an optimum for an infinite number of beams which only have one dimension, whereby the stiffness and strength are directly proportional to the weight. Furthermore, Michell does not consider buckling. Despite the theoretical nature of these structures, it is worth mentioning that they represent the only mathematical optimum known for mechanical, topological problems.

There are certain structures that meet these conditions, according to Michell. A method for deriving the structures is also given by Chan (Chan, 1960). Some of these Michell structures are shown in Figure 2. In Figure 2a), three basic forms of the main strain fields identified are shown. In Figure 2b), structures are shown which are based on these basic forms. In Figure 2c), Michell structures provided with indicated force directions and bearing positions are presented. These structures represent twodimensional solutions for topological problems. 
Michell structures have two fundamental disadvantages. Firstly, they are only defined clearly for twodimensional cases. Secondly, they only achieve optimality if some of the bars are infinitesimally thin. If there is no limitation to employing truss structures, flat structures with a variable wall thickness exhibit higher strengths with the same weight in two-dimensional cases. In three-dimensional cases, shell-shaped structures with a variable wall thickness are also stronger than truss structures of the same weight. Theoretically, an increase in strength of up to $200 \%$ is possible when employing enclosed structures, while this figure is still up to $30 \%$ in more realistic cases (Sigmund et al., 2016).

Despite these limitations, Michell structures are employed as a means of validating numerical, continuous approaches such as the SIMP approach (Sigmund et al., 2016).

a)
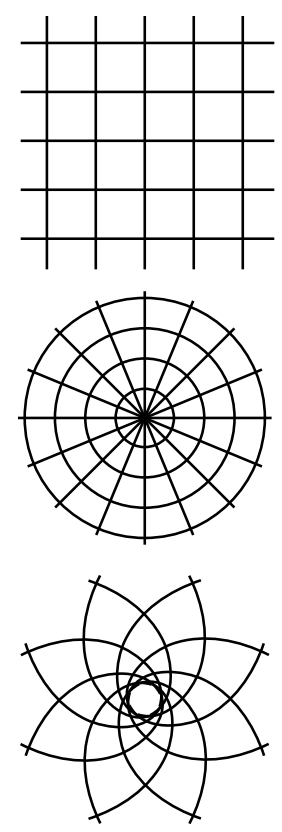

b)
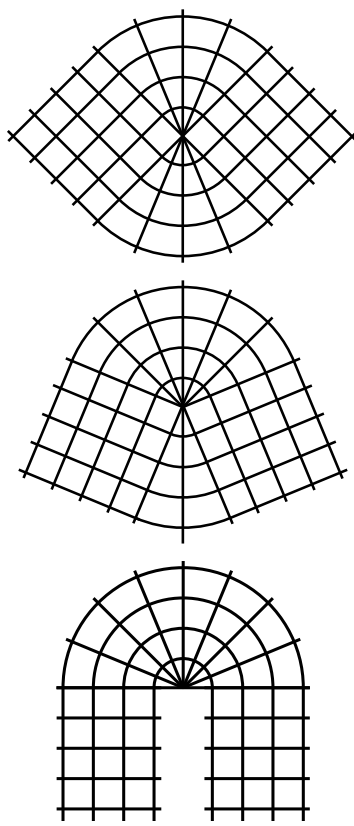

c)
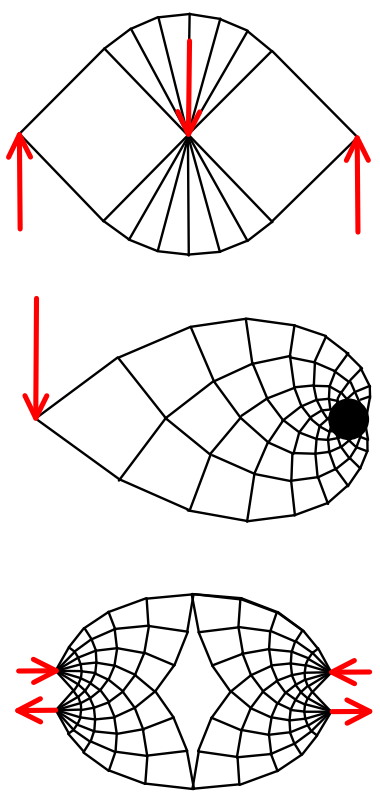

Figure 2: a) Basic forms of the main strain fields according to (Wiedemann, 2007); b) Deformation-compatible combinations according to (Wiedemann, 2007); c) Michell structures for various load cases according to (Michell, 1904)

\subsection{Mathematical approaches to structural optimization}

There are various approaches for identifying optimal structures for a given condition, namely mathematical approaches and also paper-based methods that support designers.

In continuous topology optimization, the volume elements of the assembly space to be filled with material are determined (Wiedemann, 2007). Continuous approaches to topology optimization include "Solid Isotropic Material with Penalization" (SIMP) and the "Soft Kill Option" (SKO). In the SIMP approach, the modulus of elasticity of the finite elements for a defined design space is coupled with the density of the respective element. The optimization searches for the optimal distribution of mass at a given fill level of the design space in order to keep the stiffness of the structure as high as possible. This method is therefore an optimization in a mathematical sense. Currently, most topology optimizers utilized in CAD programs utilize this SIMP approach. The SKO approach is an iterative approach. At each iteration, the modulus of elasticity of a finite element of a defined design space is set proportionally to the stress under load. This calculation principle is repeated until homogeneous material utilization has been achieved. The SKO approach thus simulates biological growth. (Harzheim, 2014)

Difficulties with these approaches are usually caused by the resolution of finite elements or non-convex problems. Resolution problems can be solved by refining the mesh and thus increasing the computing power required. It is more difficult to find a solution for non-convex problems. The algorithm does not optimize beyond a local minimum, which is not the global minimum. (Bendsøe and Sigmund, 2004)

\subsection{Paper-based optimization methods}

In addition to mathematical, computer-aided approaches to optimization, other methods allow the derivation of a suitable topology merely by using a pen and paper. The effort required to utilize these methods is lower than for the ones mentioned above, yet they also have a much lower accuracy. 
Such fast methods are advantageous during the early phases of development in particular. Drawing solutions provide a special option for conveying ideas and concepts in the early stages of development (Dylla, 1991). The utilization of these methods assists in forming a multiplicity of alternatives in order to select the most suitable one in the late concept phase and early draft phase.

Among the drawing methods are the "thinking tools after nature" (Mattheck, 2010) and some further developments based on these (Posner et al., 2014). The four main thinking tools are "tension triangles", "shear squares", "thinking in ropes" and the "force-cone method". The realization of these support methods is presented in Figure 3. The "tension triangles" method is a means of eliminating stress increases in notches or unnecessary material in corners, as shown in Figure 3a). To illustrate the results of shear, the "shear squares" method is applied. The basic consideration of this method is to illustrate the symmetry of the stress vector, as shown in Figure 3b). In order to achieve a structure which is only loaded maximally in every volume element, it is helpful to think in terms of compression bars and traction ropes. Ropes are always uniformly loaded between the forceintroduction points. Structures that can be modelled using only ropes and compression bars are thus close to an optimum. One rope structure is compared with a Michell structure in Figure 3c). The idea of the force-cone method is that, within a cone of only $90^{\circ}$, forces can be absorbed in the form of compressive and tensile forces (Mattheck, 2010). The elaboration of this method by Posner (Posner et al., 2014) introduces a space restriction for the procedure of building a topology with this idea, as shown in Figure 3d).

a)

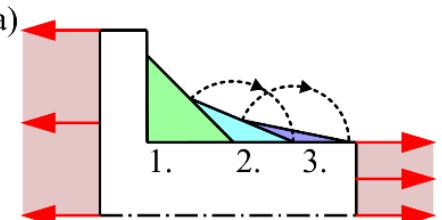

c)

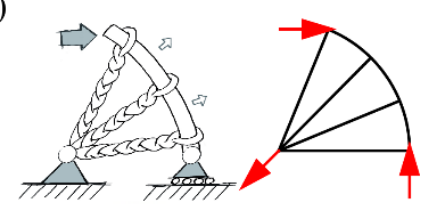

b)

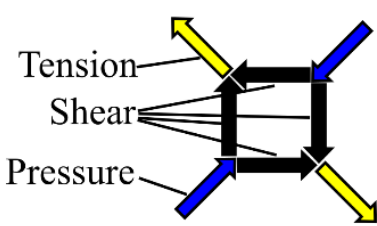

d)

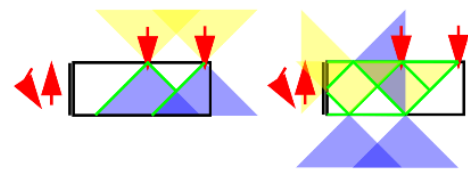

Figure 3: a) Tension triangles (modified from Sauer, 2008); b) Shear square (modified from Mattheck, 2010); c) Comparison of a rope construction (modified from Mattheck, 2010) with a Michell structure; $d$ ) The use of force cones to deduce a topology (modified from Posner et al., 2014)

\subsection{Deficits of existing approaches to the deduction of topologies}

Support in the derivation of topologies is provided in two different ways. While paper-based support methods are of use in low-detail design, mathematical support provides guidance for detailed design. During the early phases of the design process in particular, time efficiency is vital since several variants must be considered. The time required for topological optimization of the individual variants quickly adds up. Reduction of development time is a universal objective of design support methods (Pahl et al., 2007) and also advantageous in lightweight design (Keller and Binz, 2009; Posner et al., 2012). Comprehensibility is one further important measure for support. Designers have different tasks in mind and a support method which does not require full attention is beneficial (Keller and Binz, 2009; Posner et al., 2012).

Keller and Binz discuss eight important groups of requirements for engineering support methods and methodologies comprising a total of 19 elements. In the following sections of this paper, they are considered measures which can be fulfilled to a certain extent. Of these, the two most influential measures are "reliability" and "learnability". Reliability describes the extent to which the designer can trust the results of the support method employed. Conversely, learnability describes the difficulty involved in understanding and subsequently utilizing the support method.

Considering the reliability of the described support method for early stages of design, the thinking tools rely on the accuracy of the designer in deriving a topology. Mathematical support is strongly dependent on the accuracy of the input from the designer. This can lead to inaccuracies in the results, as shown in (Süß et al., 2018). The application of mathematical support methods is also hard to learn. By contrast, the thinking tools are much simpler. 
Table 1 shows a comparison of the two support strategies in the context of the early phases of design with the four measures described. The demand for a straightforward support method for the derivation of topologies is clearly visible with regard to time efficiency, comprehensibility and learnability.

Table 1: Comparison of existing support

\begin{tabular}{|c|c|c|}
\hline$\bigcirc$ Not fulfilled; $\bigcirc$ 1/2 fulfilled; $\bigcirc$ fulfilled & $\begin{array}{c}\text { Thinking tools after } \\
\text { nature }\end{array}$ & Mathematical support \\
\hline Time efficiency & (1) & O \\
\hline Comprehensibility & - & - \\
\hline Reliability & (1) & - \\
\hline Learnability & () & (1) \\
\hline
\end{tabular}

\section{A NEW APPROACH}

The measures employed to identify the deficits of the existing support methods are also requirements for newly developed support methods. The time-efficient and comprehensible use of support in other areas developed with machine learning - or artificial neural networks, to be specific - brings the application of this technology to support designers into consideration.

\subsection{Artificial neural networks}

In the conventional use of computers, an engineer writes a program, and the computer runs this program with input data to produce output results. In the application of machine learning, this principle is reversed. The engineer does not supply the computer with a program, but rather with a set of input data and assigned favorable output results called "ground truth". The computer then writes a program which can convert input to output. The process of writing the program by analyzing pairs of input and output data is referred to as "training". This reversal of principle is illustrated in Figure 4. While the training of a neural network is time-consuming and demanding in terms of computing power, the application of the trained network as a program is fast and conserves resources. (Aggarwal, 2018)

Conventional use of computers:

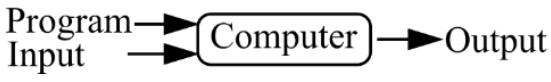

Machine learning:

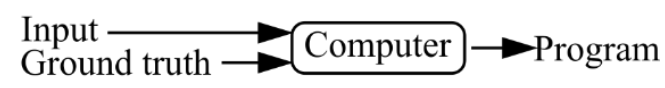

Figure 4: Comparison of traditional use of computers and machine learning

The utilization of artificial neural networks is a special kind of machine learning which is capable of processing pictures (Isola et al., 2016). Neural networks consist of several interconnected neurons or nodes. Each neuron can process incoming signals and transfer them onward. The number of consecutive neurons between the input and output is called "depth", while parallel neurons are termed a "layer". Depending on the task to be solved, different processing neurons, a different number of layers and a different number of neurons per layer are to be used. This configuration of a neural network is referred to as "architecture". (Aggarwal, 2018)

Artificial neural networks are an established IT method for pattern-recognition problems. Current research results also show ways in which these neural networks can be employed to generate patterns or images (Isola et al., 2016). An artificial neural network should therefore be trainable in order to provide a designer with the patterns to support topology derivation.

\subsection{A new procedure for deriving topologies}

The Michell structures discussed in Section 2.1 are, in theory, not dimensioned components, but the ideal force path for a given load case. These structures are patterns followed by a force path, thus a calculation of the actually prevailing forces is not necessarily needed for the generation of optimized topologies. Based solely on the direction of forces, a neural network should be able to generate a force path that the designer can use to achieve a topologically optimized design. The efficiency of trained neural networks makes it possible to support the designer with a smartphone app. The main benefits of such a method would be fast derivation of topologies in a design phase where only a sketch of the component exists. In application, it would therefore be comparable with the "thinking tools after nature". The possible use of a tool that supports this method will be demonstrated in a use case below. 
When deriving the structure for a component, the designer can use a sketch. In this sketch, the possible design space and the position of bearings and forces within this design space are outlined. One possible scenario is given in Figure 5a). In order to reduce the number of considerations for lightweight design and to concentrate completely on the functionality of the construction elements, the designer can then take a photograph of the design space as illustrated in Figure 5b). An app on the smartphone of the designer would then interpret the sketch (Figure $5 \mathrm{c}$ ) and generate the Michell structures (Figure 5d). Based on the Michell structures learned by the network, the designer could direct the forces within the component according to the optimal force paths.

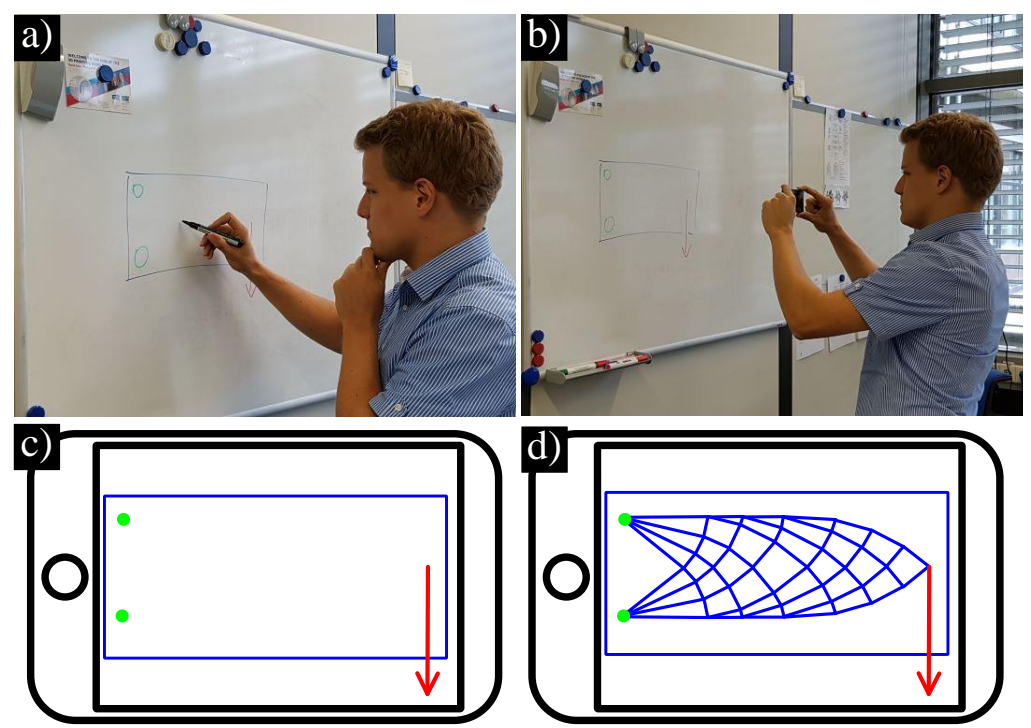

Figure 5: Procedure for utilizing the new method

This approach significantly shortens initial optimization during the concept phase. It is not necessary to create different drawings with drawn-in force cones, tension triangles or thrust squares, nor is it necessary to generate a CAD model in which forces, material and design space can be precisely defined. However, such a tool cannot replace classical approaches. Even in this fast concept on paper, the designer must estimate the quality of the results. The "thinking tools after nature" are still an effective method in this case. Computer-aided methods cannot be replaced for the dimensioning and fine optimization of a construction. Simulation is indispensable as this method cannot specify a dimension for the load-bearing structure.

\section{RESULTS OF A FEASIBILITY STUDY}

A feasibility study was carried out in order to verify the actual viability of the new method in a tool. A neural network was therefore trained to derive Michell structures for given load cases. This study did not focus on the applicability of the tool, rather on the learning ability of artificial neural networks. In Section 4.1, the architecture utilized for the network and the training of the network are described. For the actual evaluation of the method, three steps were chosen and are described in Section 4.2, while these results are discussed in Section 5.

\subsection{The neural network utilized}

In order to implement this feasibility study, a network developed for picture completion was utilized (Radford et al., 2015). This architecture is widely employed for performing image-to-image translation (Isola et al., 2016).

For the feasibility study, various Michell structures were generated based on models from (Michell, 1904) and (Chan, 1960). From 12 determined load cases, a training data set of 576 images was generated by rotating, scaling and mirroring, as well as by varying. During the modeling, a design space was defined on a black background using a blue surface. Red arrows symbolized forces and their direction. The neural network described above was trained with 400 learning cycles. One element of the training dataset is shown in Figure 6. 

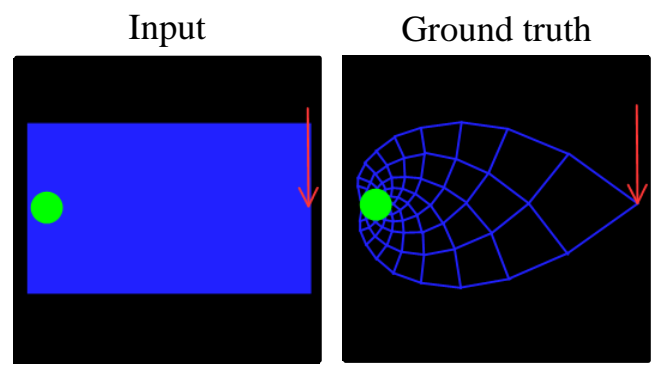

Figure 6: Sample training data of the neural network utilized

\subsection{Results of a three-stage evaluation}

To evaluate the new approach, the neural network described in Section 4.1 was given different tasks in the form of inputs. The results of the trained model - or rather the generated output - are shown and described in this section. Interpretation and conclusions are conducted and drawn separately in Section 5.

Three stages were chosen to evaluate this application. Firstly, an examination was conducted to determine how close the structures generated by the network come to the learning process. A sample evaluation is shown in Figure 7.

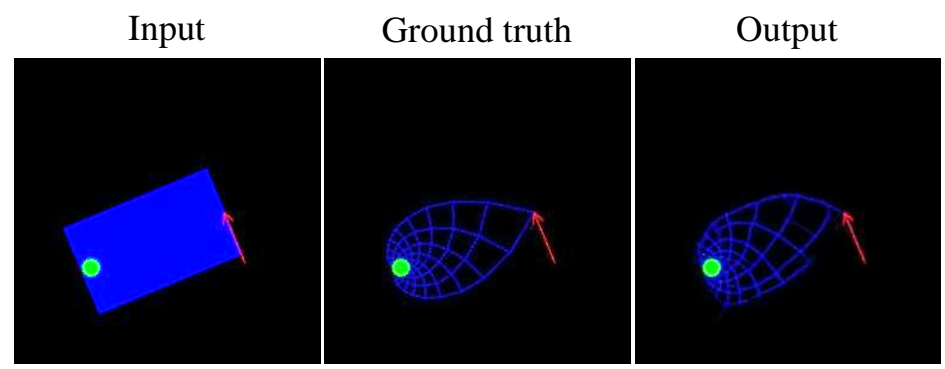

Figure 7: First evaluation of the neural network

The main difference between ground truth and output is the incompletely connected path net. The location of the bearing and the force is interpreted correctly, and the orientation of the force is correct. The shape and path of the structure are likewise correct.

In the second step of the evaluation, an example frequently shown in the context of additive manufacturing was used. In the public design challenge of General Electrics and GrabCAD (GE, 2018), an optimized design for a jet-engine bracket was created. For this task, various types of information concerning the component were given, including the construction space and load cases.

The comparison of the individual load cases and the results of the neural network and computer-aided topology optimization are summarized in Figure 8.

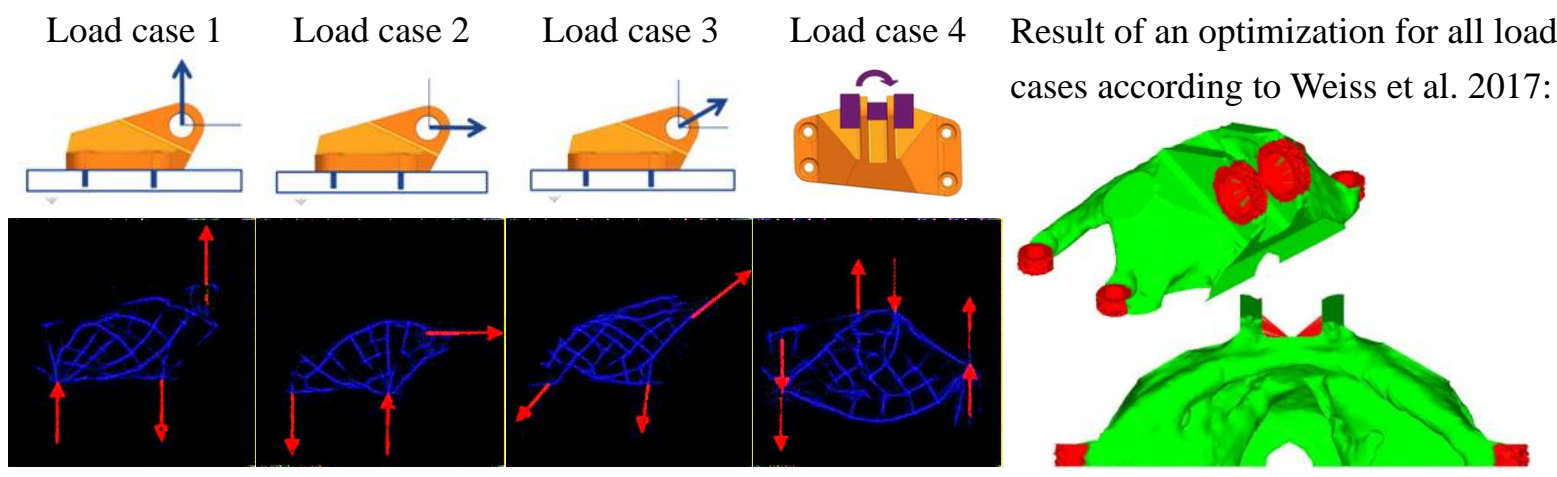

Figure 8: Comparison of the results of an optimization with the new method and a computeraided optimization according to (Weiss et al., 2017)

The new approach suggests four options; one for each load case. The results do not show completely closed force paths. The form and path of the force paths are also not completely smooth. The optimization with the SIMP method can result in an accumulated design. This is not possible for the new approach due to the underlying principle of processing one load case at a time. 
The third evaluation is a case study, for which a bottle opener was designed using the prototype of the tool. The first step defining force directions and design space, shown in Figure 9a), was performed on a computer because the network is not yet capable of processing photos. The derived topology suggestion is shown in Figure 9b). The design was created on white paper with a focus on manufacturability and usability as shown in Figure 9c). For the conversion of topology suggestions into designs, the output of the network was firstly drawn and, secondly, several force paths were chosen. The design was then modelled in polynurbs as shown in Figure 9d) and dimensioned utilizing an FEM simulation presented in Figure 9e). The printed object is shown in Figure 9f).
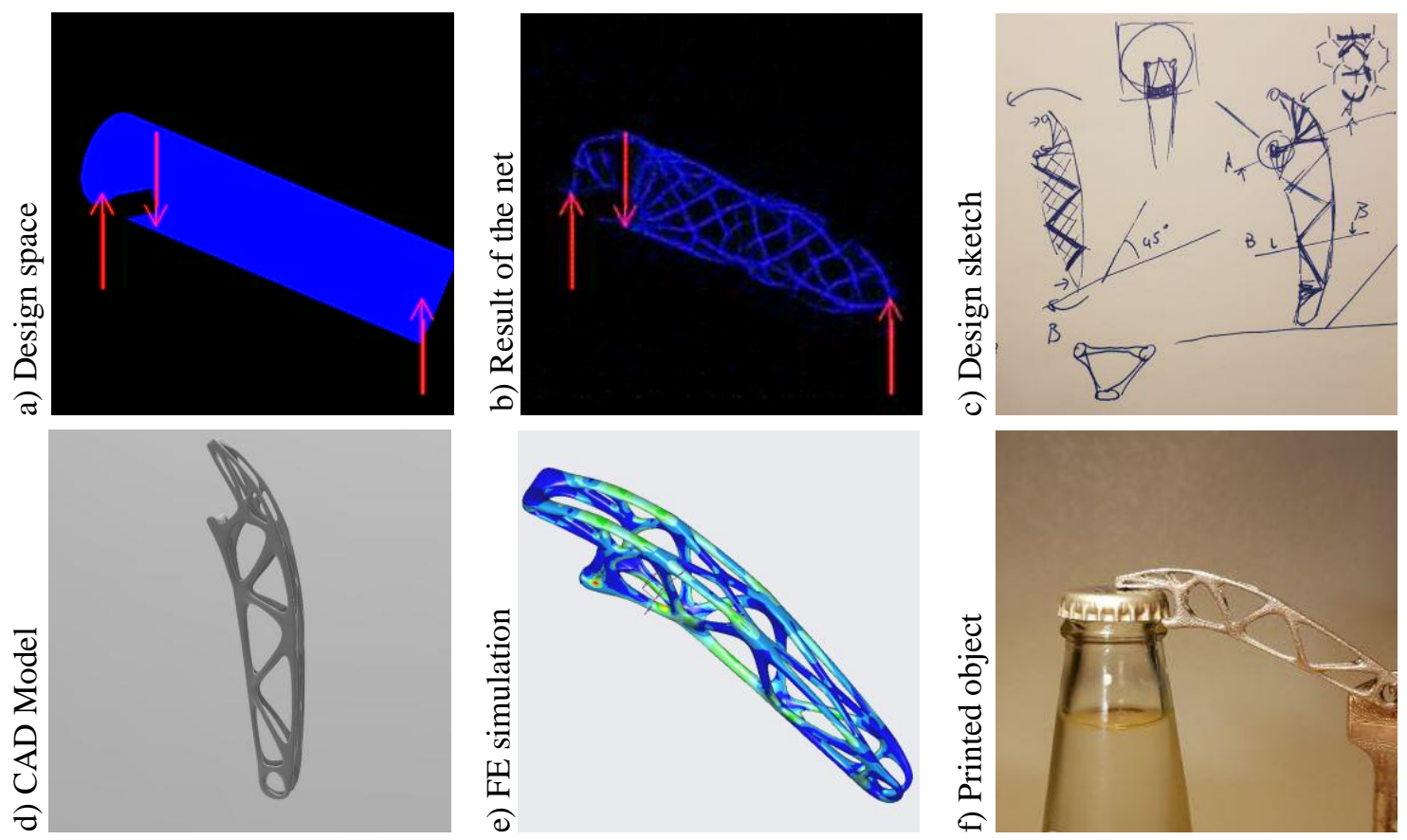

Figure 9: Design process for a bottle opener using the new design tool

\section{DISCUSSION OF THE RESULTS}

In the first stage of the evaluation, it can be seen that a neural network of the chosen structure is capable of completing the Michell structures. To ensure that the net only generates closed paths, more examples should be utilized for training. In a direct comparison with a conventional topology optimizer working with an SIMP approach, the results of the approach presented here are not very detailed. Nevertheless, the results indicate a similar direction of optimization. The practical evaluation shows that correct conclusions can be drawn from the results of the tool. The interpretation of the topology suggestion helped to design a uniformly loaded structure.

Several advantages of the new approach become apparent by applying the four criteria described in 2.4. Regarding comprehensibility, the case study demonstrated that little effort is required to interpret the results produced by the neural network. Learning the procedure for utilizing the new support method seems to be possible with just a few training sessions. The inputs and outputs are quite intuitive, while the quality of the results is lower than those of the computer-aided approaches. Nonetheless, the results are reliable. The first step of evaluation shows that the reproduction of Michell structures is repeatable and independent of orientation and scale. The time efficiency of the new support method is clearly a positive point for the new approach. In the current state, the network solves approximately 100 tasks per second. An initial design proposal can thus be generated within a very short time. This speed gives designers a considerable advantage.

\section{CONCLUSION AND FUTURE WORK}

The new method has proven its utility alongside existing support methods for deriving component topology. The greatest potential of the new support method is in its low time consumption. The new method can generate topological suggestions of medium quality in a relatively short time. However, 
designers must accept that results are of a slightly lower quality in comparison to other computer-aided methods. Nevertheless, the new method is a straightforward approach to the deduction of topologies.

In order to expand the applicability of the outlined tool, a larger catalogue of examples for training the network will be created, and a web application will be developed. Using an improved tool prototype, investigation can then be undertaken into how the new tool can be suitably utilized. Whether or not the generated topology suggestions are sufficient must also be clarified. Further it will be investigated how three-dimensional structures can be derived.

It is conceivable that there are other potential uses for this type of design-support method. With regard to additive manufacturing, an indication of excessive overhangs in sketches could also prove advantageous.

\section{REFERENCES}

acatech - Deutsche Akademie der Technikwissenschaften (2016), Additive Fertigung, München.

Aggarwal, C.C. (2018), Neural Networks and Deep Learning: A Textbook, Springer International Publishing, Cham.

Bendsøe, M.P. and Sigmund, O. (2004), Topology Optimization: Theory, Methods, and Applications, Engineering Online Library, 2nd ed., corr. printing, Springer, Berlin.

Chan (1960), The design of Michell Optimum Structures: Reports and Memoranda No. 33o3, Vol. 190, Bedford.

Dylla, N. (1991), Denk- und Handlungsabläufe beim Konstruieren, Zugl.: München, Techn. Univ., Diss., 1990, Konstruktionstechnik München, Vol. 5, Hanser, München.

GE (2018), "GE jet engine bracket challenge”, available at: https://grabcad.com/challen-ges/ge-jet-enginebracket-challenge.

Gebhardt, A. (2016), Generative Fertigungsverfahren: Additive Manufacturing und 3D-Drucken für Prototyping - Tooling - Produktion, 5., neu bearbeitete und erweiterte Auflage, Hanser, München.

Harzheim, L. (2014), Strukturoptimierung: Grundlagen und Anwendungen, 2nd ed., Verlag Europa-Lehrmittel, s.l.

Isola, P., Zhu, J.-Y., Zhou, T. and Efros, A.A. (2016), "Image-to-image translation with conditional adversarial networks".

Keller, A. and Binz, H. (2009), "Requirements on engineering design methodologies", in Bergendahl, M.N., Grimheden, M., Leifer, L., Skogstad, P. and Badke-Schaub, P. (Eds.), Proceedings of the International Conference on Engineering Design 2009, Design Theory and Research Methodology, Stanford, CA/USA, 24.-27.08.2009, Design Society, Glasgow, pp. 203-214.

Mattheck, C. (2010), Denkwerkzeuge nach der Natur, 1st ed., Karlsruher Inst. für Technologie Campus Nord, Karlsruhe.

Michell, A.G.M. (1904), "LVIII. The limits of economy of material in frame-structures", The London, Edinburgh, and Dublin Philosophical Magazine and Journal of Science, Vol. 8 No. 47, pp. 589-597.

Pahl, G., Beitz, W., Feldhusen, J. and Grote, K.-H. (2007), Konstruktionslehre: Grundlagen erfolgreicher Produktentwicklung ; Methoden und Anwendung, 7. Aufl., Springer, Berlin, Heidelberg.

Posner, B., Binz, H. and Roth, D. (2014), "Supporting the development of lightweight design structures with simple methods in early stages", DS 81: Proceedings of NordDesign 2014, Espoo, Finland, 27-29th August 2014, pp. 795-804.

Posner, B., Keller, A., Binz, H. and Roth, D. (2012), “Anforderungen an eine methode zum leichtbaugerechten konstruieren".

Radford, A., Metz, L. and Chintala, S. (2015), Unsupervised Representation Learning with Deep Convolutional Generative Adversarial Networks.

Sauer, A. (2008), “Untersuchungen zur Vereinfachung biomechanisch inspirierter Strukturoptimierung", Ph.D. thesis, 2008.

Sigmund, O., Aage, N. and Andreassen, E. (2016), “On the (non-)optimality of Michell structures", Structural and Multidisciplinary Optimization, Vol. 54 No. 2, pp. 361-373.

Süß, M., Richter, R., Hofmann, D., Schöne, C. and Stelzer, R. (2018), “eines Luftfahrtbauteils Impact of optimization target definition for maximizing the utilization of weight reduction to enhance an aircraft bracket", in Kynast, M. (Ed.), Rapid.Tech + FabCon 3.D: International Trade Show \& Conference for Additive Manufacturing, Erfurth, 05.-07.06.2018, Carl Hanser Verlag, München, pp. 65-80.

Weiss, F., Ahlgrimm, L., Binz, H. and Roth, D. (2017), "Prozess zur computergestützten Anpassung AMgerechter Lösungsprinzipien an produktspezifische Randbedingungen", in Stuttgarter Symposium für Produktentwicklung SSP 2017 Stuttgart, 29. Juni 2017, Wissenschaftliche Konferenz, Universität Stuttgart.

Wiedemann, J. (2007), Leichtbau: Elemente und Konstruktion, Klassiker der Technik, 3. Auflage, SpringerVerlag Berlin Heidelberg, Berlin, Heidelberg.

Wohlers (2017), Wohlers Report 2017: 3d Printing and Additive Manufacturing State of the Industry Annual Worldwide Progress Report, Wohlers Associates, Fort Collins, Colorado. 